\title{
Influência do treinamento da potência muscular sobre a capacidade de execução de tarefas motoras em mulheres idosas
}

\author{
Influence of muscle power training on the ability to implement motor tasks for older women
}

\section{Resumo}

Introdução: A manutenção da capacidade de geração de potência muscular é fator preponderante para a independência física e funcional do idoso. Objetivo: Avaliar o efeito de um programa de treinamento contra resistência em velocidade (PTCRV) sobre a potência muscular e o desempenho em tarefas motoras em idosas. Métodos: 58 voluntárias sedentárias e fisicamente independentes foram divididas em dois

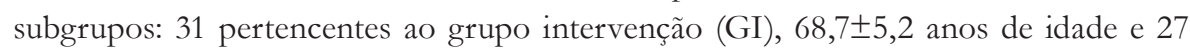

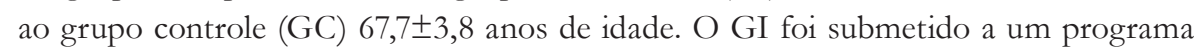
de 24 sessões, três vezes semanais, de treinamento contra resistência em velocidade. Foram avaliadas a carga (CR), a potência (PT) e a velocidade (VL) no exercício extensão de joelhos (EJ) e no teste motor de levantar-se da cadeira (LC), utilizando o sistema computadorizado Tendo Weightlifting Analyzer (TWA). Foi avaliado também o tempo de execução das tarefas motoras: marcha em velocidade de conforto (MVC) e marcha em velocidade máxima (MVM). Para comparação das variáveis estudadas, aplicou-se o teste $t$, admitindo-se $\mathrm{p}=0,05$. Resultados: Foi observado no GI para o exercício EJ ganho significativo na capacidade de geração da potência $(49,56 \pm 13,52 \mathrm{w}$ / 66,87 $\pm 16,27 \mathrm{w}, \mathrm{p}=0,0002)$. No teste $\mathrm{LC}$, houve aumento significativo na capacidade de geração da potência $(370,71 \pm 106,26 \mathrm{w} / 434,52 \pm 107,15 \mathrm{w}, \mathrm{p}=0,02185)$ e da velocidade $(0,61 \pm 0,14 \mathrm{~m} / \mathrm{s} / 0,72 \pm 0,14 \mathrm{~m} / \mathrm{s}, \mathrm{p}=0,00274)$. Nos testes motores, MVC e MVM, houve redução significativa no tempo de execução $(4,56 \pm 0,63 \mathrm{~s} / 4,20 \pm 0,50 \mathrm{~s}, \mathrm{p}=0,01560)$ e $(3,45 \pm 0,40 \mathrm{~s} / 3,23 \pm 0,34 \mathrm{~s}, \mathrm{p}=0,02222)$, respectivamente. No GC não foi observada melhora significativa em nenhum dos testes aplicados. Conclusão: O PTCRV contribuiu para melhorar os níveis de potência muscular, além de melhorar o desempenho nas tarefas motoras no grupo estudado.

Programa de Pós-graduação em Ciências Médicas, Faculdade de Ciências Médicas, Centro Biomédico. Universidade do Estado do Rio de Janeiro. Rio de Janeiro, RJ, Brasil.

Celia Cohen Barros'

Célia Pereira Caldas'

Luiz Alberto Batistal

Palavras-chave:

Envelhecimento. Exercício. Atividade Motora. Capacidade Funcional. 


\section{Abstract}

Introduction: The maintenance of the generating capacity of muscle power is an important factor for physical and functional independence of the elderly. Objective: Investigation of the influence of the training of muscular power (PTCRV) in old women during pre-determined motor chores. Methods: 58 sedentary physically independent volunteers, divided in two groups: 31 belonged to the intervention group (GI) 68.7 \pm 5.2 years and 27 to the control group (GC) $67.7 \pm 3.8$ years. The GI was subjected to a 24 -section program, three times a week, of counter-resistance training in speed. Power (PT) and speed (VL) in the exercise were assessed: knee extension (EJ), and in the stand-upfrom-a-chair test (LC), using the software Tendo Weighlifting Analyzer (TWA). The time of execution of motor chores was also evaluated: gait in comfortable speed (MVC), and gait in maximum speed (MVM). The variation $t$ test was applied to compare the studied variables and a level of significance $\mathrm{p}=0.05$ was admitted. Results: It was observed in the GI, for the EJ exercise, a significant gain in the ability of power generation (370.71 $\pm 106.26 \mathrm{w} / 434.52 \pm 107.15 \mathrm{w}, \mathrm{p}=0.02185)$. In the LC test there was significant gain in the ability of power generation $(370.71 \pm 106.26 \mathrm{w} / 434.52 \pm 107,15 \mathrm{w}, \mathrm{p}=0.02185)$ and speed $(0.61 \pm 0.14 \mathrm{~m} / \mathrm{s} / 0.72 \pm 0.14 \mathrm{~m} / \mathrm{s}, \mathrm{p}=0.00274)$. In the motor tests MVC and MVM, there was significant gain in speed $(4.56 \pm 0.63 \mathrm{~s} / 4.20 \pm 0.50 \mathrm{~s}, \mathrm{p}=0.01560)$ and $(3.45 \pm 0.40 \mathrm{~s} / 3.23 \pm 0.34 \mathrm{~s}, \mathrm{p}=0.02222)$, respectively. No significant improvement in the GC was observed after application of the tests. Conclusion: The PTCRV contributed to improve the muscular power, as well as improving the performance in the motor chores in the studied group.
Key words: Aging. Exercise. Motor Activity. Functional Ability.

\section{INTRODUÇÃO}

Conceituada como sendo uma qualidade do indivíduo em estar apto a realizar tarefas de vida diária de forma independente, a capacidade funcional inclui o conjunto de atividades de deslocamento, de autocuidado, ocupacionais e recreativas, ${ }^{1}$ dentre outras, constituindo, por isso, fator determinante da qualidade de vida das pessoas no decurso do envelhecimento.

No contexto do declínio da capacidade funcional das pessoas, a diminuição da capacidade muscular associada ao envelhecimento está diretamente relacionada ao comprometimento da capacidade de gerar força muscular. ${ }^{2}$ De forma geral, esse comprometimento se relaciona diretamente a um quadro de sarcopenia e é agravado pela limitação progressiva da mobilidade. Decorre disso um maior risco a ocorrência de quedas, maior incidência de acidentes devidos a fraqueza muscular, fadiga precoce e precárias condições de equilíbrio corporal. ${ }^{3}$ Estudos sobre treinamento de força realizados nos últimos anos têm constatado que o fortalecimento muscular melhora a qualidade do equilíbrio corporal, ${ }^{4}$ diminui o risco de quedas e, consequentemente, de fraturas ósseas. ${ }^{5}$

Em termos específicos, por meio do fortalecimento muscular, pretende-se capacitar o músculo para distintos tipos de ações, dentre elas, as de geração de força máxima, endurance e potência. A potência muscular é derivada da força muscular e está relacionada com a capacidade de produzir tensão rapidamente. ${ }^{6}$ Tal capacidade é importante em ações que visam responder com rapidez e eficácia às diferentes tarefas motoras impostas ao sujeito no dia a dia, sendo determinante da magnitude do tempo de reação e do tempo de movimento em ações motoras diversas. Assim a disfunção dessa capacidade, por parte do indivíduo, dificulta a realização de respostas motoras eficazes, notadamente em situações de perigo, como são as que envolvem perda súbita do equilíbrio. ${ }^{1}$

Há evidências de que, com o avanço da idade, a capacidade de gerar potência declina em taxa mais pronunciada que a capacidade de gerar força 
máxima, e também de que o primeiro fenômeno se mostra mais fortemente associado com a perda da capacidade funcional que o segundo. ${ }^{3} \mathrm{E}$ possível então inferir que não basta simplesmente garantir a manutenção da capacidade de gerar força muscular; mais que isso, é preciso garantir que a força seja gerada de forma rápida, ou seja, é preciso garantir a manutenção de bons níveis de capacidade de gerar potência muscular. A redução da capacidade de gerar potência muscular implica também uma redução de velocidade da contração dos músculos esqueléticos, agravando o estado do quadro de mobilidade corporal do idoso. ${ }^{7,8}$

Pelo fato de esse complexo processo de declínio aumentar a taxa de perda progressiva da independência física e funcional, no decorrer do envelhecimento, é importante promover o desenvolvimento de estratégias que visem minimizar os efeitos do envelhecimento muscular. Dentre as estratégias atualmente utilizadas, encontram-se aquelas voltadas ao denominado "treinamento da potência muscular", as quais têm-se mostrado eficazes na redução da taxa de avanço da instalação do quadro acima descrito. ${ }^{7-11}$

Neste estudo objetivou-se avaliar, em mulheres idosas, o efeito de um programa de atividades físicas focado no treinamento contra resistência em velocidade (PTCRV) sobre a potência gerada por músculos de membros inferiores, mensurada no decurso da execução de duas tarefas motoras distintas: extensão simultânea de joelhos (EJ) e levantar-se da cadeira (LC), e sobre a magnitude da velocidade de deslocamento, mensurada em duas situações distintas de marcha.

\section{METODOLOGIA}

Trata-se de estudo de intervenção estruturado em três etapas: testagem prévia, intervenção e testagem posterior; e pelo exame de dois grupos distintos, um que sofreu intervenção (GI) e o outro de controle (GC).

A amostra, estruturada por conveniência, foi composta por 58 voluntárias, frequentadoras do Centro de Convivência da Universidade Aberta da Terceira Idade na Universidade do Estado do Rio de Janeiro (UnATI/UERJ), entre dezembro de 2005 e julho de 2008, tendo sido considerados os seguintes critérios de inclusão: ter idade igual ou superior a 60 anos de idade; ser fisicamente independente; nunca ter participado de programas de exercícios físicos de força do tipo contra resistência e ser capaz de realizar todos os exercícios e testes propostos. Foram considerados critérios de exclusão: ser portadora de doenças articulares ou musculares, deficiências físicas ou mentais e doenças cardíacas. As inicialmente selecionadas foram divididas, aleatoriamente, em dois grupos, GI e GC. No final do experimento, o GI foi constituído por 31 indivíduos com idade média de $68,7( \pm 5,2)$ anos, e o GC por 27 indivíduos com idade média de $67,7( \pm 3,8)$ anos.

O projeto deste estudo foi aprovado pelo Comitê de Ética em Pesquisa (CEP) do Hospital Universitário Pedro Ernesto da Universidade do Estado do Rio de Janeiro (parecer no 1413CEP/HUPE). Após serem informadas sobre todos os procedimentos adotados na pesquisa, as voluntárias que concordaram em participar do estudo assinaram o Termo de Consentimento Livre e Esclarecido.

Todas as integrantes dos dois grupos passaram pela mesma rotina de testes, sendo ambos os grupos submetidos a duas mensurações, uma antes da intervenção (pré) e a outra após os dois meses durante os quais essa transcorreu (pós).

As componentes do GC foram orientadas a manter suas atividades cotidianas normais e a não participar de nenhum tipo de treinamento que envolvesse exercícios de força muscular com pesos durante o período de dois meses. Já o GI foi submetido ao PTCRV, no qual foram realizadas 24 sessões de exercício, com frequência de três vezes semanais, em dias alternados, perfazendo um período total de aproximadamente dois meses.

Para cada exercício do PTCRV, foram realizadas três séries de oito repetições com cargas individualizadas, executadas com a maior velocidade possível na sua fase concêntrica. Oito exercícios compuseram o PTCRV: leg press; flexão de joelhos na posição de pé; extensão simultânea 
de joelhos; abdução de quadril; adução de quadril; rosca tríceps, executados em máquinas da marca Righetto; rosca bíceps e elevação lateral dos membros superiores executados com pesos livres. A resistência utilizada no treinamento foi equivalente a $80 \%$ do valor de resistência associado à melhor curva obtida no teste da potência.

Durante o período de treinamento, foram feitos três ajustes nas cargas, com o propósito de fazer com que os exercícios fossem realizados por maior período de tempo no transcurso do programa, com uma carga que representasse $80 \%$ da potência manifestada em cada instante temporal.

Para avaliação da velocidade de movimentos das voluntárias, foram utilizados testes motores de marcha e levantar-se da cadeira, integrantes da bateria de testes de Williams \& Greene, descritos por Matsudo. ${ }^{12}$ As medidas de força e potência muscular foram realizadas por meio do método de exame da curva de potência, utilizando-se o equipamento Tendo Weightfting Analyzer - modelo V104 (TWA), segundo estratégia validada por Jennings \& Viljoen. ${ }^{13}$

Antes da realização dos exames, todas as voluntárias foram submetidas a uma preparação prévia para os testes que iriam ser executados, pois foi constatado que a não familiarização com os exercícios constituiria variável interveniente importante, prejudicando a qualidade da mensuração e, consequentemente, dos resultados dos testes. Foram marcados três encontros para a realização dos testes.

A mensuração da massa corporal das voluntárias foi realizada por meio de uma balança eletrônica digital da marca Filizola ${ }^{\circledR}$ (Brasil), previamente calibrada. A variável "massa corporal" foi monitorada durante o estudo.

Examinou-se o tempo de execução da marcha em duas modalidades de marcha diferenciadas entre si quanto à magnitude da velocidade de deslocamento: marcha em velocidade de conforto (MVC), considerada aquela que a pessoa utiliza normalmente no dia a dia para locomover-se de um ponto a outro, e a marcha em velocidade máxima (MVM), aquela na qual o sujeito se desloca tão rápido quanto lhe é possível, conservando o padrão biomecânico de deslocamento em marcha.

Para medir a MVC, foi demarcada no chão uma faixa com 30 centímetros de largura e três metros de comprimento. As voluntárias foram orientadas a percorrer o trajeto demarcado, caminhando na velocidade em que normalmente se deslocam no dia a dia, sem correr ou afastarse da trajetória predeterminada, sendo admitido algum nível de desvio lateral de forma que o padrão de marcha habitual não tivesse que ser alterado. O cronômetro era acionado na voz de comando inicial e parado quando o último pé ultrapassava a linha de chegada demarcada no chão, distante três metros do ponto de partida. As participantes examinadas foram orientadas a não sair do percurso e a continuar andando mesmo depois de ultrapassar a linha de chegada, evitando que começassem a reduzir a velocidade quando estivessem próximas ao final do percurso. Para a mensuração da variável MVM, foram adotados os mesmos procedimentos descritos para o teste de MVC, sendo que as voluntárias foram solicitadas a percorrer a distância de três metros com a máxima velocidade de deslocamento que conseguissem implementar, sem correr.

$\mathrm{Na}$ mensuração da velocidade ao levantar da cadeira (LC), foi utilizado um dispositivo constituído pelo equipamento TWA, um banco de madeira com $43 \mathrm{~cm}$ de altura, base com $45 / 45 \mathrm{~cm}$ e microcomputador da marca Toshiba ${ }^{\circledR}$ A10-S169. Para cada teste realizado, o aparelho de medida foi ajustado de acordo com a massa corporal da voluntária em exame, a qual era orientada a permanecer sentada na posição padronizada para o teste até que recebesse a ordem para realizar a tarefa motora. No início da etapa de mensuração, as voluntárias respondiam ao comando de partida, levantando-se do assento na maior velocidade possível, passando para a posição de pé. Cada voluntária realizou três tentativas, sendo utilizado, dentre as três, a de resultado com maior valor de potência. 
Para a mensuração da potência dos músculos extensores do joelho, utilizou-se o exercício de extensão simultânea de joelhos (EJ), realizado em uma cadeira extensora da marca Righetto. A resistência inicial foi determinada por ensaio e erro. A partir de um valor inicial, a resistência foi incrementada progressivamente até que se identificasse a carga com a qual a voluntária conseguisse gerar a maior média de potência registrada pelo TWA. Entre uma tentativa e outra, estabeleceu-se um tempo de cinco minutos de repouso, de forma a coibir a influência da fadiga muscular sobre o resultado do teste. Para controle da influência do grau de resistência passiva inerente ao equipamento utilizado, todos os testes, para todas as voluntárias, foram realizados no mesmo aparelho, tendo sido o mesmo previamente lubrificado.

Uma vez identificada a resistência relativa à máxima potência, outra visita ao laboratório era agendada, na qual a voluntária realizava três repetições do exercício EJ, sendo adotado como resultado final o registro da repetição em que a voluntária alcançasse sua melhor média de potência.

Para análise estatística foram avaliados os dois grupos. Em cada um deles foram mensuradas a potência (PT) e a velocidade (VL) no exercício extensão de joelhos (EJ), e no teste motor de levantar-se da cadeira (LC). Foi avaliado também o tempo de execução das tarefas motoras: marcha em velocidade de conforto (MVC) e marcha em velocidade máxima (MVM).

As análises estatísticas foram feitas em duas etapas. $\mathrm{Na}$ primeira foram confrontadas, para todos os testes, as avaliações pré e pós em cada um dos grupos separadamente, GC e GI (comparação intragrupos). Na segunda etapa, foram confrontadas entre os dois grupos, GC e GI, as diferenças dos resultados obtidos na primeira etapa das análises (comparação intergrupos). Para essas comparações, foram utilizados o teste $t$ e o nível de significância de referência alfa foi de $5 \%$.

\section{RESULTADOS}

Os resultados serão apresentados conforme as etapas descritas na metodologia.

\section{$1^{a}$ etapa: Avaliação comparativa entre os testes pré e pós- PTCRV para GC e Gl}

Após a análise descritiva para todos os testes nos grupos GC e GI, os ganhos obtidos foram comparados para avaliar o efeito do treinamento em cada um dos grupos (avaliação intragrupos).

\section{Teste da Extensão do Joelho (EJ)}

Para EJ, foram medidas as variáveis velocidade $\mathrm{m} / \mathrm{s}$ (VL) e potência W (PT). Pode-se inferir que no GC não ocorreram mudanças significativas na VL e na PT entre as avaliações pré e pós. Para o GI, o PTCRV provocou aumento significativo na capacidade de produção de potência muscular, mas o mesmo não se pode afirmar para a variável VL. Os valores destes achados estão descritos na tabela 1.

Tabela 1 - Ganhos médios estimados e nível de significância para os testes de velocidade (m/s) e potência (W) na extensão de joelhos (EJ). Rio de Janeiro-RJ, 2011.

\begin{tabular}{lcccc}
\hline & Velocidade $(\mathrm{m} / \mathrm{s})$ & $\mathrm{p}$ & Potência $(\mathrm{W})$ & $\mathrm{p}$ \\
\hline Grupo controle & $-0,01556$ & 0,923 & $-1,29630$ & 0,890 \\
Grupo intervenção & $-0,00129$ & 0,534 & 18,06452 & $1,54 \mathrm{e}-09$ \\
\hline
\end{tabular}




\section{Teste de Levantar-se da Cadeira (LC)}

Para o LC foram medidas as variáveis velocidade $\mathrm{m} / \mathrm{s}(\mathrm{VL})$ e potência $\mathrm{W}(\mathrm{PT})$. Podese inferir que para o GC nas variáveis VL e PT não houve modificação significativa entre as avaliações pré e pós. Para o GI, há evidências de que o TCRV provocou aumento significativo tanto para velocidade do movimento quanto para a capacidade de produção de potência muscular. Os valores desses achados estão descritos na tabela 2 .

Tabela 2 - Ganhos médios estimados e nível de significância para os testes de velocidade $(\mathrm{m} / \mathrm{s})$ e potência (W) no levantar-se da cadeira (LC). Rio de Janeiro-RJ, 2011.

\begin{tabular}{lcccc}
\hline & Velocidade $(\mathrm{m} / \mathrm{s})$ & $\mathrm{p}$ & Potência $(\mathrm{W})$ & $\mathrm{p}$ \\
\hline Grupo controle & $-0,057$ & 0,943 & $-38,148$ & 0,9983 \\
Grupo intervenção & 0,108 & $2,24 \mathrm{e}-06$ & 63,806 & $6,73 \mathrm{e}-06$ \\
\hline
\end{tabular}

\section{Teste Motor Marcha em Velocidade de Conforto (MVC) e Marcha em Velocidade Máxima (MVM)}

Para os testes motores MVC e MVM, foram medidos os tempos de execução da tarefa em segundos. Com base nos resultados encontrados, pode-se afirmar que o GC apresentou diferença significativa entre as avaliações pré e pós da MVC, mas para a MVM o mesmo não ocorreu, pois a mudança não foi significativa. Para o GI, há evidências para afirmar que o PTCRV provocou redução significativa do tempo de execução das tarefas avaliadas. As evidências no grupo GI são mais fortes que no grupo GC, vide os valores de p. Os resultados são apresentados na tabela 3.

Tabela 3 - Ganhos médios estimados no tempo de execução em segundos e nível de significância para os testes motores marcha em velocidade de conforto (MVC) e marcha em velocidade máxima (MVM). Rio de Janeiro-RJ, 2011.

\begin{tabular}{lcccc}
\hline & MVC (s) & $\mathrm{p}$ & MVM (s) & $\mathrm{p}$ \\
\hline Grupo controle & $-0,1738$ & 0,03443 & $-0,0354$ & 0,242 \\
Grupo intervenção & $-0,3013$ & 0,01137 & $-0,2171$ & 0,00168 \\
\hline
\end{tabular}

\section{$2^{a}$ etapa: Comparação entre os ganhos de GC e Gl}

Nesta etapa foram comparados os ganhos médios para cada um dos testes confrontando os dois grupos GC e GI (avaliação intergrupos).
O resultado para o teste extensão de joelho (EJ) na variável velocidade (VL) não foi significativo $(p=0,44)$, ou seja, não houve evidências estatísticas para afirmar que o ganho médio do GC $(0,015 \mathrm{~m} / \mathrm{s})$ diferiu do ganho médio do GI $(-0,001)$. 


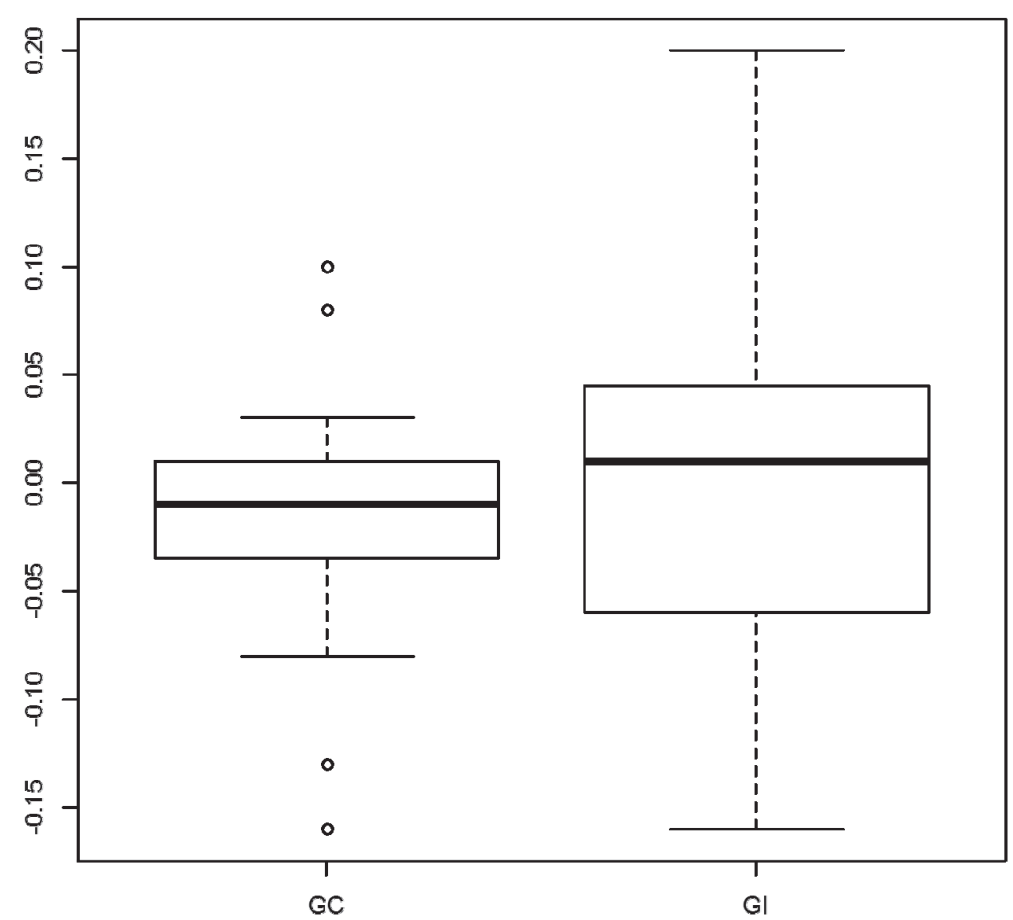

Figura 1 - Comparação entre ganhos dos GC e GI para o teste EJ velocidade m/s. Rio de Janeiro-RJ, 2011.

Para o teste EJ na variável potência (PT), houve evidências estatísticas para afirmar que o ganho médio do GC (-1,30W) diferiu do ganho médio do GI (18,1W). O GI mostrou aumento da capacidade de produção de potência muscular significativamente maior que o $\mathrm{GC}(\mathrm{p}=4,84 \mathrm{e}-10)$.

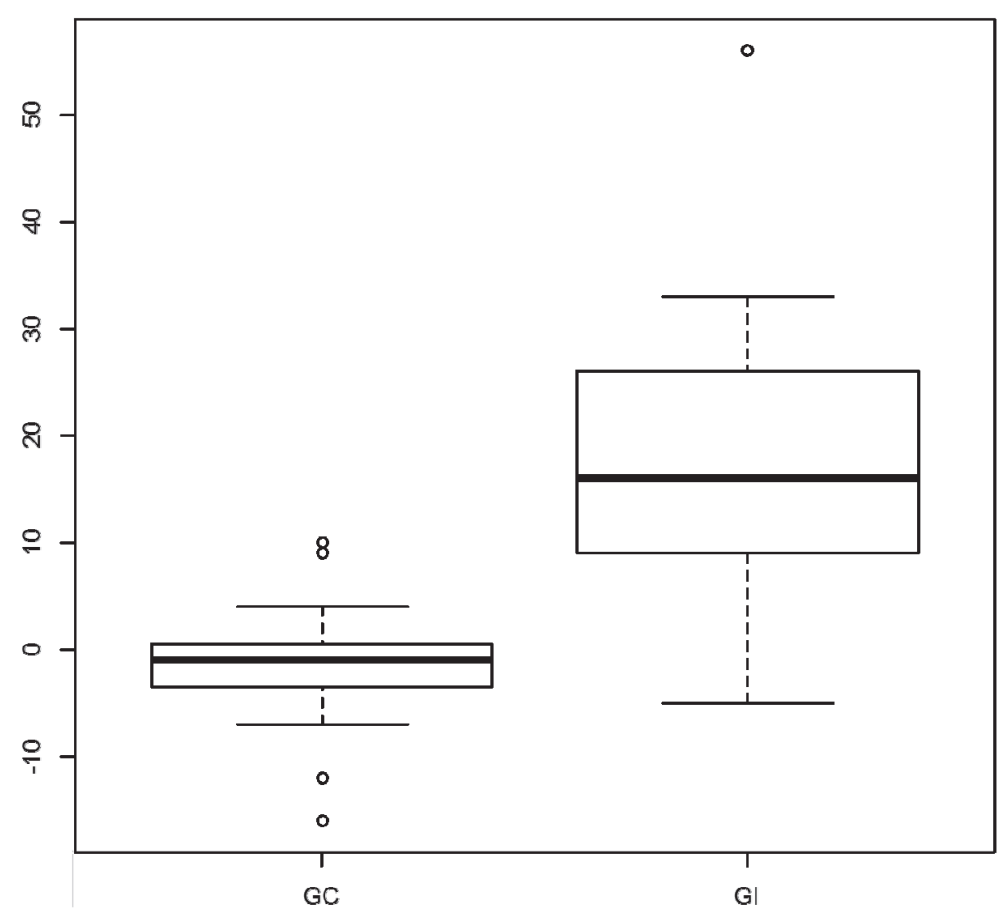

Figura 2 - Comparação GC e GI para o teste EJ variável potência W. Rio de Janeiro-RJ, 2011. 
Para o teste LC na variável velocidade (VL), afirmar que o GI mostrou aumento $(0,108 \mathrm{~m} / \mathrm{s})$ houve evidências estatísticas $(\mathrm{p}=5,94 \mathrm{e}-07)$ para significativamente maior que o GC $(-0,057 \mathrm{~m} / \mathrm{s})$.

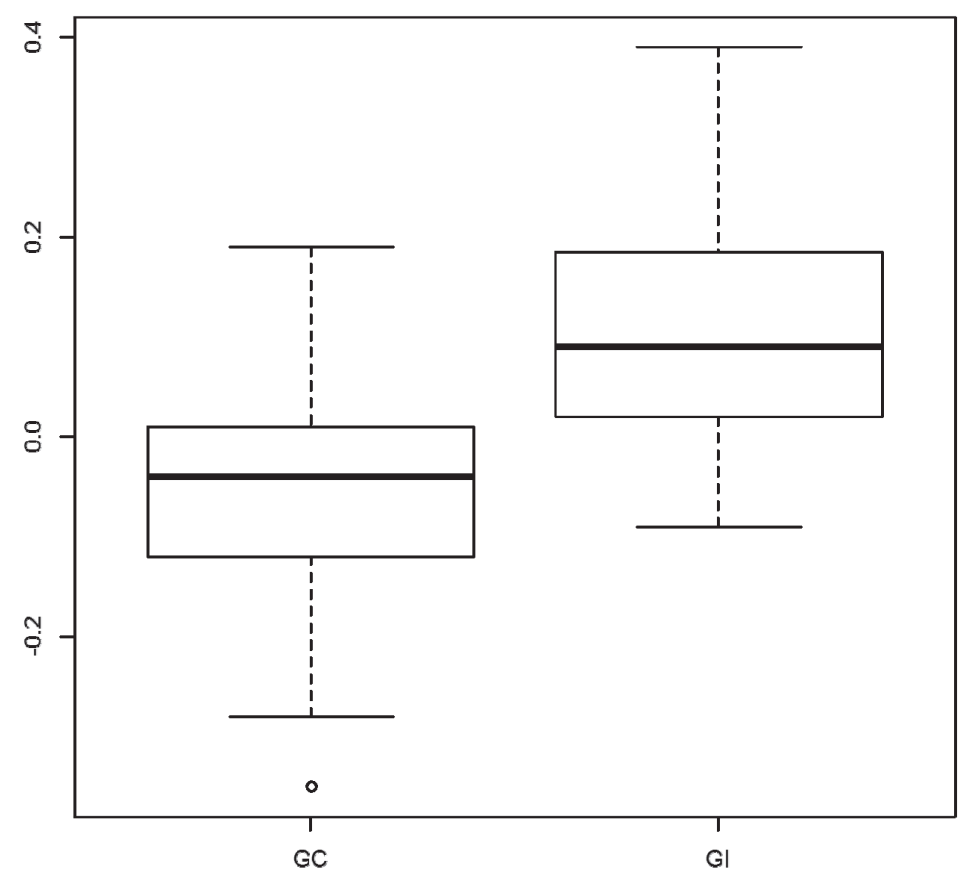

Figura 3 - Comparação GC e GI para o teste LC velocidade m/s. Rio de Janeiro-RJ, 2011.

Para o teste LC na variável potência (PT), houve evidências estatísticas $(\mathrm{p}=1,22 \mathrm{e}-05)$ para afirmar que o GI mostrou um ganho positivo $(68,8 \mathrm{~W})$ significativamente maior que o GC $(-38,1 \mathrm{~W})$.

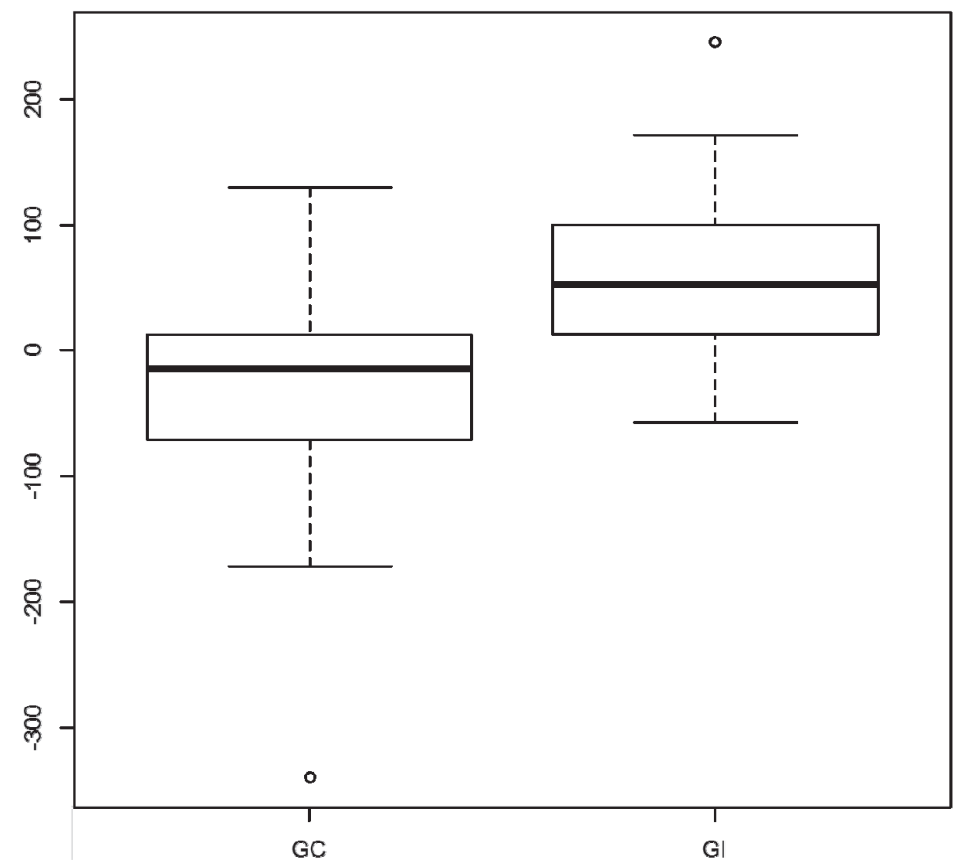

Figura 4 - Comparação GC e GI para o teste LC potência W. Rio de Janeiro-RJ, 2011. 
Para o teste Marcha em Velocidade de Conforto (MVC), não houve diferença significativa estre os grupos GC $(-0,174 \mathrm{~s})$ e GI

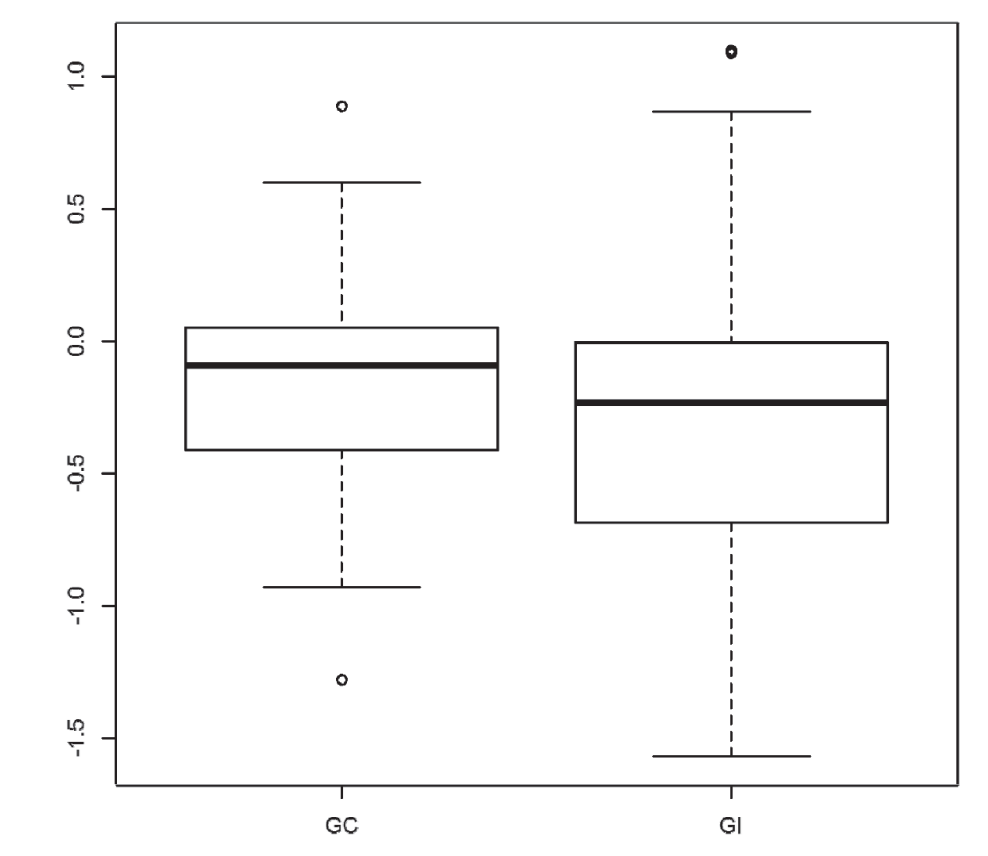

Figura 5 - Comparação dos ganhos entre GC e GI para o teste MVC. Rio de Janeiro-RJ, 2011.

Para o teste MVM, há evidências para afirmar que houve diferença significativa $(\mathrm{p}=0,035)$ entre os grupos GC e GI. Observando os resultados na (-0,301s), apesar de o GI ter apresentado redução significativa no tempo de execução da tarefa.

snificativis

os
figura 6, o GI mostrou redução no tempo (-0,217s) para execução da tarefa significativamente maior que GC (-0,0354s).

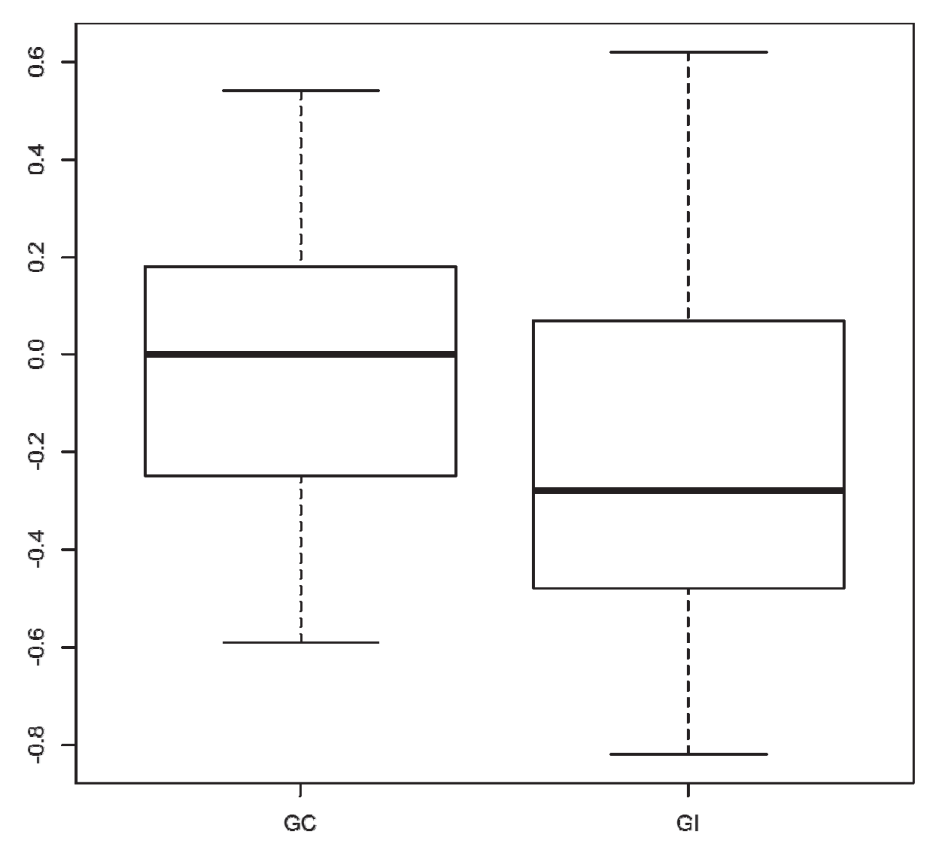

Figura 6 - Comparação dos ganhos entre GC e GI para o teste MVM. Rio de Janeiro- RJ, 2011. 


\section{DISCUSSÃO}

A maior parte dos resultados obtidos nesta pesquisa ratifica os achados de outros estudos científicos, que embora tenham utilizado metodologias diferentes, tiveram objetivos semelhantes, confirmando a eficácia do treinamento de potência para pessoas idosas.- ${ }^{7-9,11,14}$

Os resultados obtidos indicam, para o grupo de intervenção, aumento significativo da resistência após o período de treinamento no exercício extensão de joelhos. Este achado sugere que houve aumento na capacidade de geração da força, uma vez que foram verificados aumentos expressivos nos valores da resistência infligidos às voluntárias do grupo de intervenção. Resultados similares foram obtidos pelos pesquisadores Botaro et al., ${ }^{15}$ Earles et al., ${ }^{16}$ Fielding et al., ${ }^{14}$ De Vos et al. ${ }^{17}$ e Hruda et al. ${ }^{18}$

Quanto à velocidade dos movimentos, verificou-se que não houve aumento significativo no exercício de extensão de joelhos. É importante ressaltar, no entanto, que mesmo com o aumento expressivo da resistência, também não houve redução da velocidade. Tal fato demonstra melhora no desempenho do movimento, tendo em vista a relação inversa entre a resistência e a velocidade. ${ }^{19}$ Nos testes motores, os resultados demonstraram ganhos significativos na velocidade de movimento no teste de levantarse da cadeira e, ainda, redução significativa do tempo de execução nos testes da marcha resultados que também foram verificados por Bean et al. ${ }^{20}$ e Hazellet al. ${ }^{21}$

Observou-se, neste estudo, ganho significativo na capacidade de geração da potência muscular, verificado por meio do equipamento Tendo Weightlifiting Analyzer V-104, no exercício extensão de joelhos e no teste de levantar-se da cadeira.
$\mathrm{Na}$ EJ, o aumento na capacidade de geração da potência muscular se deu principalmente pelo aumento das resistências deslocadas, e não pelo aumento da velocidade desenvolvida no movimento. Já no teste de levantar-se da cadeira, o ganho na capacidade de geração da potência se deu pelo aumento na velocidade do movimento, uma vez que a resistência deslocada pelas voluntárias era a própria massa corporal, a qual não sofreu modificações significativas durante $o$ tempo de intervenção.

Deve-se considerar como limitação deste estudo a ausência de equipamento para uniformização da velocidade dos movimentos durante as sessões de treinamento e de um protocolo validado de treinamento da potência muscular.

\section{CONCLUSÃO}

O programa de treinamento contra resistência em velocidade (PTCRV) na metodologia descrita neste estudo resultou no aumento da capacidade de geração da potência e da força no exercício extensão de joelhos, além de melhorar a capacidade de geração da potência e da velocidade de execução em todas as tarefas motoras examinadas. O treinamento da potência muscular, portanto, contribuiu para a melhora do desempenho nas tarefas motoras no grupo de idosas pesquisadas.

Os resultados corroboram o que é descrito na literatura, ratificando o pressuposto de que o treinamento da potência muscular em idosos pode constituir importante meio de intervenção para atenuar os efeitos negativos do declínio da capacidade funcional que cursa com $\mathrm{O}$ envelhecimento. É, assim, uma importante estratégia a ser utilizada para proporcionar melhor qualidade de vida aos idosos. 


\section{REFERÊNCIAS}

1. Ueno LM. A influência da atividade física na capacidade funcional: envelhecimento. Rev Bras Ativ Fis Saúde 1999;4(1):57-68.

2. Izquierdo M, Häkkinen K, Ilbañez J, Garrues M, Antón A, Zúñiga A, et al. Effects of strength training on muscle power and serum hormones in middle-aged and older men. J Appl Physiol 2001;90(4):1497-507.

3. Macardle W, Katch FI, Katch VL. Fisiologia do exercício: energia, nutrição e desempenho humano. Rio de Janeiro: Guanabara Koogan; 2003.

4. Bean JF, Herman S, Kiely DK, Frey IC, Leveille SG, Fielding RA, et al. Increased velocity exercise specific to Task (InVEST) training: a pilot study exploring effects on leg power, balance, and mobility in community-dwelling older women. J Am Geriatr Soc 2004;52(5):799-804.

5. Orr R, de Vos NJ, Singh NA, Ross DA, Stavrinos TM, Fiatarone-Singh MA. Power training improves balance in healthy older adults. J Gerontol A Biol Sci Med Sci 2006;61(1):78-85.

6. Bompa TO. Treinamento de potência para o esporte: pliometria para o desenvolvimento máximo de potência. São Paulo: Phorte; 2004.

7. Edström E, Altun M, Bergman E, Johnson H, Kullberg S, Ramírez-León V, et al. Factors contributing to neuromuscular impairment and sarcopenia during aging. Physiol Behav 2007;92(12):129-35.

8. Thomas DR. Loss of skeletal muscle mass in aging: examining the relationship of starvation, sarcopenia and cachexia. Clin Nutr 2007;26(4):389-99.

9. Miszko TA, Cress ME, Slade JM, Covey CJ, Agrawal SK, Doerr CE. Effect of strength and power training on physical function in community-dwelling older adults. J Gerontol A Biol Sci Med Sci 2003;58(2):171-5.

10. Serra Rexach JA. Clinical consequences of sarcopenia. Nutr Hosp 2006;21(Suppl 3):46-50.
11. Porter MM. Power training for older adults. App Physiol Nutr Metab 2006;31(2):87-94.

12. Matsudo SMM. Avaliação do idoso: física \& funcional. Londrina: Midiograf; 2000.

13. Jennings CL, Viljoen W, Durandt J, Lambert MI. The reliability of the Fitro Dyne as a measure of muscle power. J Strength Cond Res 2005;19(4):859-63.

14. Fielding RA, LeBrasseur NK, Cuoco A, Bean J, Mizer K, Fiatarone Singht MA. High-velocity resistence training increases skeletal muscle peak power in older women. J Am Geriatr Soc 2002;50(4):655-62.

15. Bottaro M, Machado SN, Nogueira W, Scales $\mathrm{R}$, Veloso J. Effect of high versus low-velocity resistance training on muscular fitness and functional performence in older men. Eur J Appl Physiol 2007;99(3):257-64.

16. Earles DR, Judge JO, Gunnarsson OT. Velocity training induces power-specific adaptations in highly functioning older adults. Arch Phys Med Rehabil 2001;82(7):872-8.

17. de Vos NJ, Singh NA, Ross DA, Stavrinos TM, Orr R, Fiatarone Singh MA.Optimal load for increasing muscle power during explosive resistance training in older adults. J Gerontol A Biol Sci Med Sci 2005;60(5):638-47.

18. Hruda KV, Hicks AL, McCartney N. Training for muscle power in older adults: effects on functional abilities. Can J Appl Physiol 2003;28(2):178-89.

19. Kraemer WJ, Newton RU. Training for muscular power. Phys Med Rehabil Clin N Am 2000;11(2):341-68.

20. Bean JF, Kiely DK, Alian J, Frontera WR. Is stair power a clinically relevant measure of leg power impairments in at-risk older adults? Arch Phys Med Rehabil 2007;88(5):604-9.

21. Hazell T, Kenno K, Jakobi J. Functional benefit of power training for older adults. J Aging Phys Act 2007;15(3):349-59. 